\title{
ON THE HIGH INTENSITY ASPECTS OF AGS BOOSTER PROTON OPERATION*
}

\author{
R.K. Reece, L.A. Ahrens, E.J. Bleser, J.M. Brennan, C. Gardner, J. W. Glenn, T. Roser, \\ Y. Shoji, ${ }^{1}$ W. vanAsselt, W.T. Weng \\ AGS Department, Brookhaven National Laboratory \\ Upton, NY 11973
}

\section{SUMMARY}

Observations of high intensity effects on the proton performance of the AGS Booster are presented, including present operational limits and correction methods. The transverse emittances, optimum tune working points, damping of coherent transverse oscillations and correction of stopband resonances through third-order are discussed in addition to the observed tune spread due to space charge forces. The initial longitudinal phase space distribution, capture and acceleration parameters and measurements are also given. Operational tools and strategies relevant to the high intensity setup are mentioned.

\section{OVERVIEW OF ACCELERATOR}

The present high intensity proton mode of operation of the Booster synchrotron is as a rapid cycling $(5 \mathrm{~Hz})$ machine which accepts the $200 \mathrm{MeV}$ Linac beam and delivers a nominal $1.5 \mathrm{GeV}$ beam to the AGS accelerator. The Booster circumference was designed to be $1 / 4$ that of the AGS so that four Booster cycles fill the AGS. By requiring the extraction rf frequency of the Booster to be the same as the injection rf frequency of the AGS (with the ratio of harmonic numbers = $1 / 4$ ), precise bunch to bucket transfer is achieved. Details of early commissioning have been given in previous proceedings $[1,2]$.

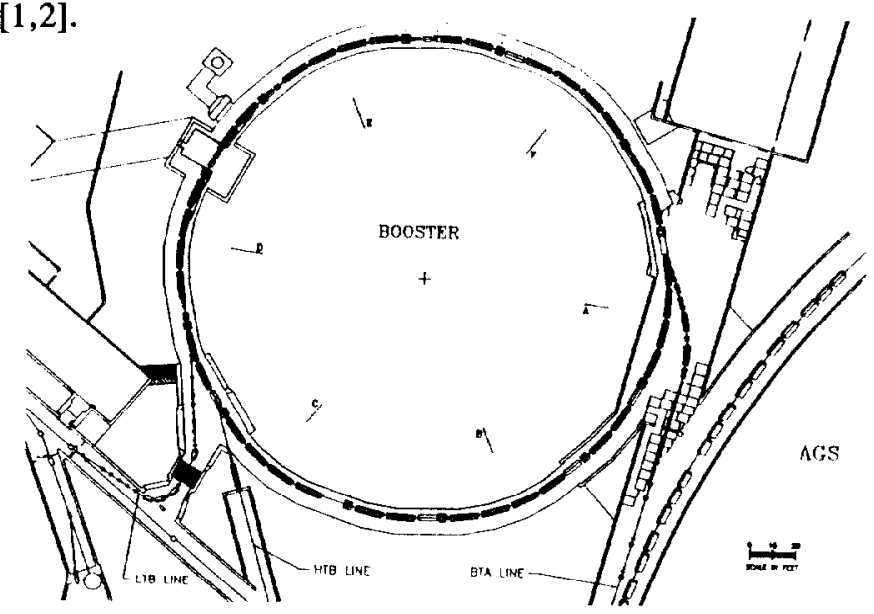

Figure 1. Booster layout.

\footnotetext{
*Work performed under the auspices of the U.S. Dept. of Energy.

${ }^{1}$ Permanent address, KEK.
}

\section{INJECTION STRATEGIES}

Injection of the Linac $\mathrm{H}^{-}$beam is accomplished using a $200 \mu \mathrm{g}$ carbon stripping foil in the Booster vacuum chamber. This foil is located to the inside (small radius) of the machine. The beam from the Linac is initially matched to the Booster lattice and steered onto the locally distorted equilibrium orbit at the foil using the transfer line magnetic elements. The transverse emittance roughly doubles during this process which leaves it still small $(12 \pi \mathrm{mm}-\mathrm{mr}, 95 \%$ normalized) relative to the Booster aperture $(80 \pi \mathrm{mm}-\mathrm{mr})$ and also relative to the emittance needed for high intensity acceleration.

The local orbit distortion is obtained using four fast injection ring magnets that allow orthogonal time-dependent position and angle control of the equilibrium orbit at the injection point. This bump is used to compensate for the $-12.3 \mathrm{~mm}$ radial motion of the orbit at the foil during the $250 \mu \mathrm{s}$ injection period caused by the $3 \mathrm{~T} / \mathrm{sec} \mathrm{dB} / \mathrm{dt}$ at injection, and to relax the orbit away from the foil after injection. An additional slow outward bump assures that the equilibrium orbit is completely off the foil once the fast bump has collapsed.

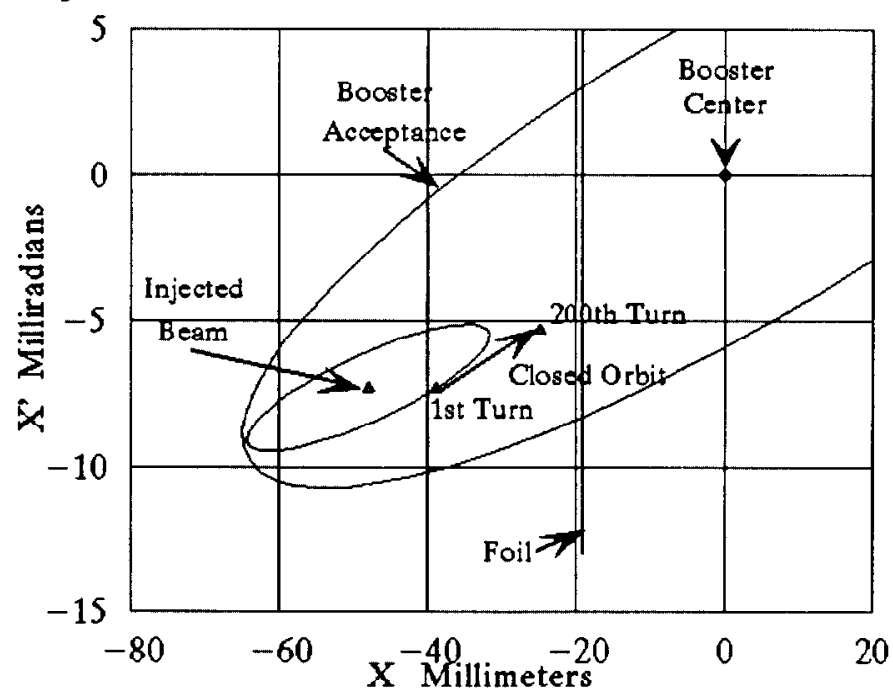

Figure 2. Booster injection phase space showing the component of closed orbit motion due to the fast injection bumps.

In order to minimize the emittance growth due to multiple passes through the foil, the fast bump is trimmed to offset the equilibrium orbit slightly toward the outside edge of 
the foil (relative to the incoming beam). This process was quantified using absolute injected beam position from a PUE located immediately downstream of the foil, and trajectory (relative to the equilibrium orbit) information from the on-line analysis of digitized multiturn data from a second PUE [3]. Both of these measurement techniques have continued to give valuable benchmarks for operations in maintaining stable injection conditions.

\section{LONGITUDINAL CONSIDERATIONS}

The rf cycle begins with bunch-to-bucket injection of the fast chopped Linac beam into a moving $1.4 \mathrm{eV}$-s bucket at $3 \mathrm{~T} / \mathrm{sec}$ with $90 \mathrm{kV}$ of $\mathrm{rf}$ voltage. The energy distribution of the $200 \mathrm{MeV} \mathrm{H}^{-}$Linac beam was measured by bunch rotation in a mis-matched bucket to be $\Delta p / p= \pm 3 \times 10^{-3}$ for $90 \%$ of the beam. This energy spread, together with the change in synchronous energy of the moving bucket for the $250 \mu \mathrm{s}$ injection pulse length is approximately equal to the bucket height. The result is an essentially uniformly filled bucket, leading to a bunching factor (average/peak current) of 0.47 . Figure 3 shows a mountain range oscillograph of a longitudinal pickup for the first $3 \mathrm{~ms}$ of the cycle. Using the maximum available of voltage at injection alleviates beam loading problems and provides a capture efficiency of $>80 \%$.

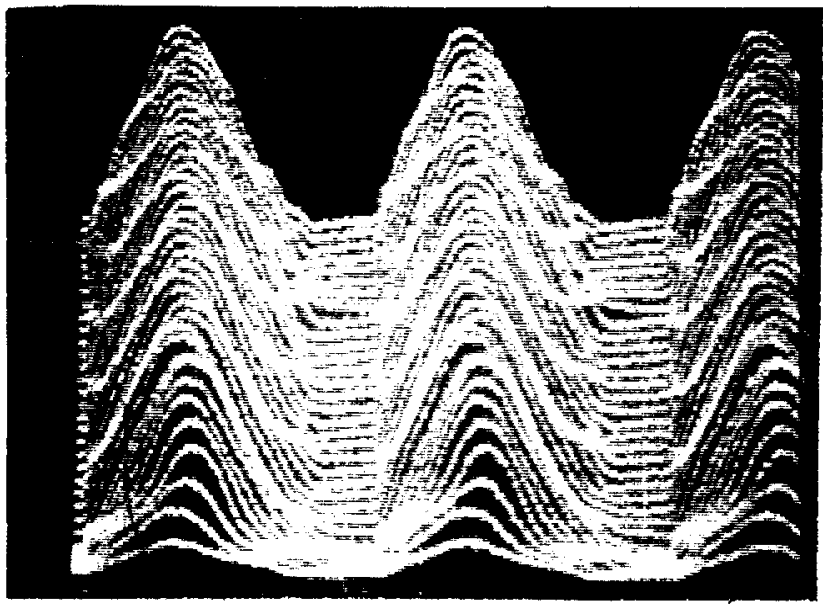

Figure 3. Mountain range through the capture process.

\section{TRANSVERSE CORRECTION}

Much of the control of the Booster machine parameters is done from a high level perspective. The main dipoles and quadrupoles are powered in series from the Main Magnet power supply. Bare machine functions (e.g., tune and chromaticity) have been measured as a function of main field and $\mathrm{dB} / \mathrm{dt}$. Variations of the bare tunes simply scale with $(\mathrm{dB} / \mathrm{dt}) / \mathrm{B}$. An overlay of incremental tune and chromaticity can be mapped onto these bare machine functions allowing the user to deal only with the final tune. The uncorrected machine closed orbit is nearly field independent. Correction of the transverse closed orbit distortions is accomplished by measuring the orbit harmonics (primarily 4th, 5th and 6th) and correcting them at one momentum, usually at or near injection. These corrections are then scaled programmatically with the main guide field $B(t)$ function to extrapolate and correct throughout the cycle.

Second and third order stopband corrections have been successfully parameterized as simple functions of $\mathrm{B}, \mathrm{dB} / \mathrm{dt}$ and radial position through extensive study with low intensity beams on main magnet cycles tailored to give a variety of time dependences[4]. This work also identified two potentially important third order lines driven by skew sextupole fields which were not originally correctable, but for which corrections have been added to the lattice, allowing the stopbands to be efficiently compensated for.

The injection $\mathrm{dB} / \mathrm{dt}$ was increased to $3 \mathrm{~T} / \mathrm{sec}$ (a factor of five over that used last year) and the transition to full $\mathrm{dB} / \mathrm{dt}$ $(7 T / \mathrm{sec})$ was shortened to reduce the time spent at lower energies (largest tune spreads) in order to reduce the impact of the peripheral stopbands on the beam.

\section{HIGH INTENSITY TECHNIQUES}

For high intensity operation, the transverse aperture of the machine is fully utilized at injection. The differentiation of beam loss due to the transverse and longitudinal techniques invoked to get to high intensity (e.g., fast bump program, if voltage and radial steering programs) from losses driven by the high intensity itself (e.g., stopband losses far from the operating point, rf beam loading) is critical in refining these techniques. An invaluable aid in defining this separation is the $750 \mathrm{keV}$ chopper [5], the primary function of which is to "chop" the Linac beam "in time" to fit into the Booster RF buckets. This chopper can also eliminate a precise fraction of this pre-bunched beam altogether and therefore reduce the intensity while preserving the overall time development of the injection process. In this mode, the rf chopper behaves like a "longitudinal sieve". See Figure 4. By reducing the normal 200 turn injection to 20 dilute turns, non-intensity dependent beam loss sources were cleanly identified. Alternately, this rf chopper permits injection of a few turns at any time during the injection interval which then allows observation of the survival of that part of the process; again, in the absence of high intensity effects.

With the tools available, it has been possible to define operationally starting values for the injection trajectory, injection field, injection fast bump program, and $\mathrm{rf}$ capture parameters independent of intensity measurements. Stopband corrections were also defined from low intensity studies. These setups have required little adjustment when intensity optimization is added as a constraint.

Referring again to the injection trajectory match, experiments are in progress to explore the intentional mismatch of the injection and equilibrium orbits, leading to a known transverse dilution or "smoke ring". While this is expected to be more effective in the vertical plane, it has been applied to the horizontal plane as a by-product of reducing foil losses. Documentation of the beam size throughout the cycle 
was done using the Ionization Profile Monitor (IPM), and a low intensity benchmark was established to be compared to in the high intensity cases. Preliminary results indicate that although the initial emittance is larger with the "smoke ring" configuration, emittance growth at high intensities may be less and lead to a net smaller emittance at extraction.
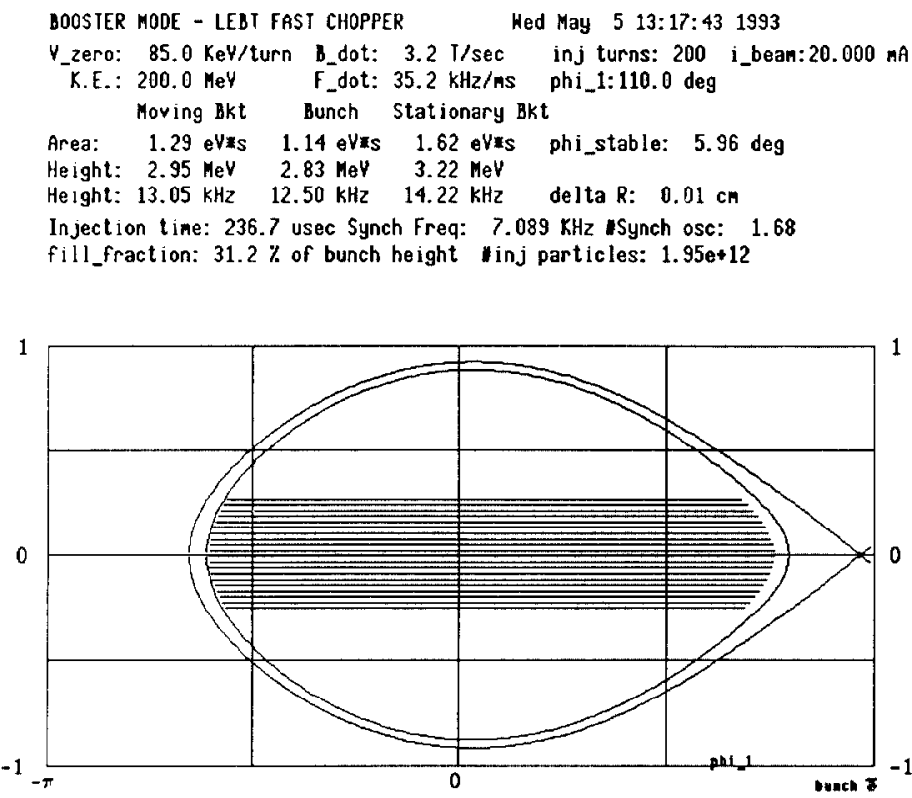

Figure 4. Longitudinal phase space: "dilute" population of 20 turns injected over the period of 200 turns.

The ability to damp transverse coherent instabilities has been incorporated into the Booster and is discussed elsewhere in these proceedings [6]. First evidence of these instabilities has been seen at an intensity of 5-7TP with the vertical chromaticity set to -0.25 and a vertical tune of 4.94 . At this intensity, the instability was eliminated by decreasing the chromaticity toward -1 .

With an injected beam intensity of $1.5 \times 10^{13}$ protons, $9 \times 10^{12}$ protons have been accelerated to extraction energy. To achieve this intensity it was necessary to move the vertical betatron tune up to near the integer $(\mathrm{Qv}=4.94)$ during the early part of the acceleration cycle (bare machine tunes are $\mathrm{Q}_{\mathrm{H}, \mathrm{V}} \approx 4.6$ ). A calculation of the incoherent tune spread using the relation below [7] yields a value of 0.3 with the following parameters; $r_{p}=1.535 \mathrm{E}-18 \mathrm{~m}, \mathrm{~N}=9 \mathrm{E} 12$ protons, $\epsilon_{N}(95 \%)=60 \pi \mathrm{mm}-\mathrm{mr}, \beta=0.5662, \gamma=1.2132$ and $\mathrm{B}$ (bunching factor) $=0.47$. It is assumed for this estimate that the beam is round and has a Gaussian density distribution where the emittance enclosed $95 \%$ of the beam.

$$
\Delta Q=\frac{3 r_{p} N}{2 \pi \epsilon_{N}(95 \%) \beta \gamma B}
$$

Given the nominal working point and that most of the 2nd and 3 rd order stopbands in the operating quadrant of tune space are corrected (linear coupling is small and corrected), and that there was an uncorrected stopband at $\mathrm{Qv}=4.67$, beam loss at intensities greater than $9 \times 10^{12}$ protons is consistent with that expected from the incoherent tune spread of this high intensity beam.

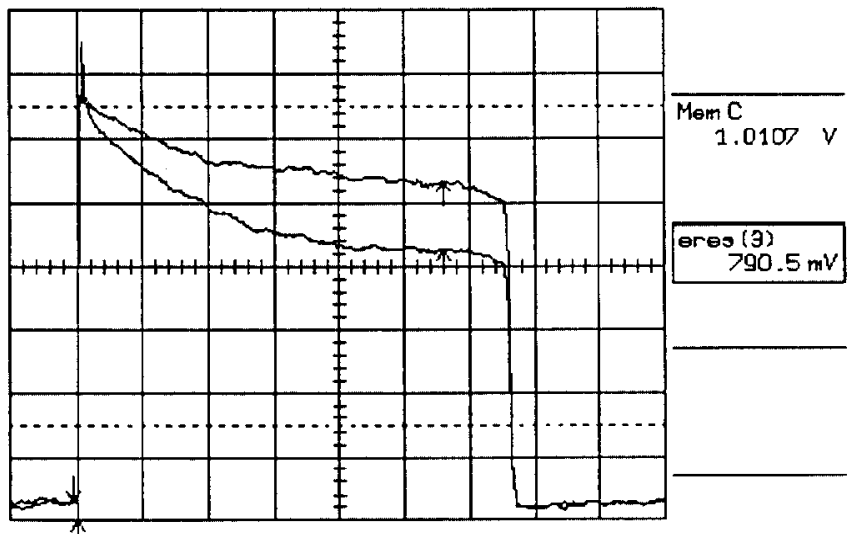

Figure 5. Circulating beam intensity $(10 \mathrm{~ms} / \mathrm{div}$.$) with$ and without a particular stopband correction applied. Upper trace yields 9.6 TP late in the cycle.

\section{ACKNOWLEDGMENTS}

The progress made in improving our understanding of the high intensity Booster came as a result of more than two months of totally dedicated study time this spring. The AGS Operations Group under A. McNerney and P. Ingrassia participated actively in the study work itself, in preparing and maintaining the machines, an in coping with the safety environment. The continuing enthusiastic involvement of several of the Booster designers and builders including $G$. Danby, Y.Y. Lee, and A. Soukas provided ideas and solutions as problems became defined. The entire exercise was only possible due to the administrative and scientific support of the AGS Department Chairman D. Lowenstein.

\section{REFERENCES}

[1] L. Ahrens, et al., "The Operational Status of the Booster Injector for the AGS Accelerator Complex at BNL', XVth Int. Conf. on H.E. Accel., Hamburg, Germany, July 1992, pp. 109-111.

[2] W.T. Weng, "Operation of the Brookhaven AGS with the Booster", these proceedings.

[3] C. Gardner, L. Ahrens, "A Method for Determining the Position, Angle and Other Injection Parameters of a Short Pulsed Beam in the Brookhaven AGS", 1985 IEEE PAC Conf. Proc. pp. 1888-1890.

[4] C. Gardner, et al., "Observation and Correction of Resonance Stopbands in the AGS Booster", these proceedings.

[5] J.M. Brennan, et al., "A Fast Chopper for Programmed Population of the Longitudinal Phase Space of the AGS", 1989 IEEE PAC Conf. Proc., pp. 1154-1156.

[6] D. Russo, et al., "Results from the AGS Booster Transverse Damper", these proceedings.

[7] L.J. Laslett, "On Intensity Limitations Imposed by Transverse Space Charge Effects in Circular Particle Accelerators", BNL7534 (1963). 